$$
\therefore \times-615-67-377
$$

$$
\text { NASA TM } \mathrm{X}=559 / 7
$$

\title{
DEMODULATION OF
}

AMPLITUDE MODULATED R.F. WAVES

IN A PLASMA AT RESONANCE:

NOBUKI KAWASHIMA

NASA

AUGUST 1967

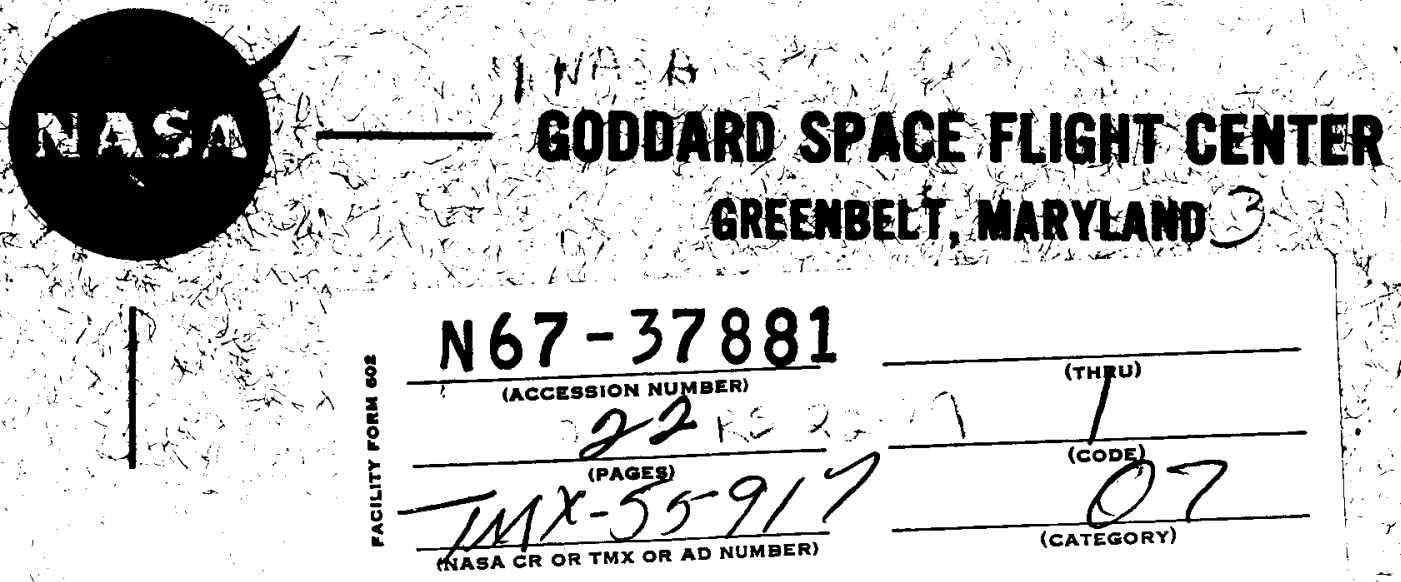




\title{
DEMODULATION OF AMPLITUDE MODULATED R.F. WAVES
}

\section{IN A PLASMa at RESONANCE}

\author{
by \\ NOBUKI KAWASHIMA* \\ Laboratory for Space Sciences \\ NASA-Goddard Space Flight Center \\ Greenbe1t, Maryland
}

\begin{abstract}
A laboratory simulation experiment was performed to study the mechanism of the detection of audio waves from nearby radio broadcasting stations by spacecraft-borne audio detectors in the ionosphere, where the carrier frequencies of the detected radio stations seem to depend on the altitude of space probes within the ionosphere. Two electrodes were immersed in a low density plasma, and an RF wave amplitude-modulated by an audio wave was fed to one of them, while the other electrode was used to detect the audio wave. The detection of the audio wave is due to the rectification and integration by the sheath around the electrode (antenna), which has a non-1inear voltage-current characteristic. The dependence of the carrier frequency on the altitude is explained as a result of series resonance of an inductive plasma with the sheath capacitance. This is just the same as the ordinary resonance probe and the resonance frequency is slightly smaller than the plasma frequency. For a higher modulation frequency, the audio output has a phase lag due to the integration by a high plasma and sheath impedance. The audio output has a strong dependence on the potential of the receiving
\end{abstract}

* NAS-NAE resident research associate on leave from the Japan Atomic Energy Research Institute, Ibaraki, Japan 
electrode (antenna) with respect to the plasma, and it reverses the polarity at the potential where the second derivative with respect to the voltage of the voltage-current characteristic of the receiving electrode in plasma is equal to zero. For higher potentials, the amplitude of the audio output shows a large broad maximum, and more than an order of magnitude larger output can be obtained than that with the ordinary resonance probe. 


\section{INTRODUCT ION}

Some recent rocket and satellite experiments show the detection of audio waves from nearby broadcasting stations by space-borne audio detectors in the ionosphere, and indicate that the carrier frequencies of the detected audio waves seem to have a dependence on the altitude (Ungstrup, 1967; Maynard, 1967). RF waves from radio broadcasting stations have a carrier frequency $\left(\omega_{1}\right)$ amplitude-modulated by audio waves $\left(\omega_{2}\right)$. In free spaces it is composed of waves of frequencies $w_{1}, w_{1}+w_{2}$ and $w_{1}-w_{2}$, but no wave of audio frequency $w_{2}$ is present. In a plasma, however, non-linear effects can be expected, especially at resonances, so that there is a possibility of the appearance of the wave of frequency $\omega_{2}$, as a result of non-linear coupling of these three waves. This experiment was performed to simulate these phenomena and clarify their physical mechanism.

The interaction of RF waves with plasma has been extensively studied by many workers, and some are utilized for the measurement of various parameters of either laboratory or ionospheric plasma. Among them, the resonance probe method, developed by Takayama et al (1960), is a useful technique for the measurement of the density of low density plasmas. The mechanism of the resonance probe is explained by the series resonance of the plasma sheath capacitance and the inductance of plasma itself near the plasma frequency, and the RF wave is rectified by the non-linearity of the sheath (Butler et al, 1963; Dote et al, 1965). Though the resonance does not take place exactly at the plasma frequency, it is a useful tool for the measurement of plasma density. In this paper it will be shown that the mechanism of audio wave detection from radio broadcasting stations in the ionosphere is the same as the resonance probe, and moreover, this experiment offers a useful technique for plasma diagnostics which has several advantages over the ordinary resonance probe from a practical point of view. 


\section{RESULTS OF EXPERIMENT}

The experimental arrangement is shown schematically in Figure 1. A plasma is produced by an RF discharge $(f \simeq 20 \mathrm{Mc} / \mathrm{s})$ in the upper part of the vacuum chamber, and a quiet plasma diffuses down to the experimental region through an RF shielding screen. A uniform plasma of the density of the order of $10^{6} / \mathrm{cm}^{3}$ is formed in the chamber of $50 \mathrm{~cm}$ in diameter and $30 \mathrm{~cm}$ in height. The RF shield is quite effective so that the RF wave for the plasma production does not interfere with the experiment. The chamber is evacuated down to $10^{-7} \mathrm{mmHg}$ and a gas of either Helium, Argon or air is filled there in the range of $10^{-4} \mathrm{mmHg}$. An amplitude-modulated $R F$ wave is fed into the plasma through an electrode $\mathrm{P}_{1}$ (25 $\mathrm{mm}$ in diameter), which corresponds to the antenna of the radio broadcasting station on the ground, and the audio wave is detected by a second electrode $\mathrm{P}_{2}$ of the same size. The output from the second electrode is fed either to an oscilloscope or to a lock-in amplifier. Figure 2 shows the oscilloscope trace of the output from the receiving electrode $\mathrm{P}_{2}$, and also shown is the input wave form of the amplitude modulated RF wave. The amplitude of the output signal is strongly dependent on the carrier frequency, and it has a large peak value for the carrier frequency near the plasma frequency (Figure 3 ).

In general, the audio output from the receiving electrode is not strictly in phase with the modulation of RF wave, and there is a certain phase difference between them. (Figure 2).

When the potential of the electrode is nearly at the plasma potential, the audio output from the electrode $P_{2}$ is $180^{\circ}$ out of phase to the modulation for a lower frequency of modulation, and a relative phase departure from $180^{\circ}$ appears when the modulation frequency is increased. The amplitude and the relative phase lag from $180^{\circ}$ as a function of modulation frequency are shown in Figure 4. The phase lag increases and the amplitude 
decreases as the modulation frequency is increased. This phenomenon is strongly dependent on the external circuit elements. When the output is terminated by an additional resistor, both the phase lag and the amplitude decrease as the resistor is decreased (Figure 5). If it is terminated by a capacitor, the phase lag increases and the amplitude decreases. The frequency for the peak audio output is compared with the resonance frequency of the ordinary resonance probe, where the receiving electrode $\mathbf{P}_{2}$ is used as a resonance probe, and it is found that the former is a little higher than the latter as shown in Figure 6.

Though an RF wave amplitude-modulated by an audio wave has in general no Fourier analysed audio component in free space, an audio component appears if there is a non-linear circuit element in the system considered. When we look at the circuit shown in Figure 1 more carefully, the electrodes $\mathbf{P}_{1}, \mathbf{P}_{2}$ and ground form a closed circuit through the plasma and the external circuit. When the receiving electrode $P_{2}$ is used an an ordinary resonance probe, the probe $P_{2}$ and the ground form a closed circuit through the plasma and the external circuit. These two circuits resemble each other quite well. In the ordinary resonance probe the probe is set nearly at space potential, and a large DC current flows in the circuit when the inductive plasma (i.e. the dielectric constant $\epsilon=1-\left(\frac{{ }^{\omega}}{\omega}\right)^{2}<0$ ) and the capacitive sheath surrounding the probe form a series resonance circuit. This is understood that the RF wave in the plasma is very large at this series resonance, and this RF wave is rectified by the non-linear voltage-current characteristic of the sheath. The output is further integrated, since the time constant of the circuit is much greater than the period of the RF wave. The same phenomenon can be expected to happen in the configuration shown in Figure 1 . The RF wave amplitude in the plasma is largest at the frequency which corresponds to a series resonance of inductive plasma with two 
sheaths surrounding the probes. In this case, the rectified and integrated output has two components; one is a DC component and the other is the audio component of modulation. Since we are looking for only the audio component, it displays a peak near at the plasma frequency. The reason why the frequency for the maximum audio output in the circuit shown in Figure 1 appears at a frequency higher than the resonance frequency of the ordinary resonance probe (when the probe $P_{2}$ is used as a resonance probe) is that there is an additional series sheath capacitance in the former circuit. However, the frequency for the maximum audio output is still lower than the plasma frequency of the plasma, which is determined from a minumum in the resonance probe characteristic (Dote et al, 1965). The phase difference between the audio output and the modulation and also its dependence on external circuit elements can simply be explained by the integration of the output in the integration circuit consisting of the effective resistance through the plasma and sheath and the stray capacitance of the external circuit. Usually, the output from the receiving electrode is fed into the input terminal of an oscilloscope preamplifier, which is equivalent to terminating the output with a $1 \mathrm{M} \Omega$ resistor and a $47 \mathrm{pF}$ capacitor. The output coaxial cable has a much higher capacitance and the total stray capacitance $C$ is about $150 \mathrm{pF}$. The effective resistance $\mathrm{R}$ through the plasma and the sheath is about $500 \mathrm{k} \Omega$, which means that the integration condition of $\omega C R=1$ is satisfied for the frequency of modulation $f=\frac{\omega}{2_{\pi}}$ of $2.1 \mathrm{kc} / \mathrm{s}$. This well agrees with the experimental result shown in Figure 4 , where the phase shift of $45^{\circ}$ (which means $\omega C R=1$ ) corresponds to the frequency of $2 \mathrm{kc} / \mathrm{s}$. Both the decrease of the phase shift and the amplitude by the termination with an additional resistor and the increase of the phase shift with an additional capacitor also confirm the interpretation described above. 
The audio output from the probe $P_{2}$ is strongly dependent on the probe potential. When the probe-to-ground potential is below $10 \mathrm{~V}$, the audio output is out of phase to the modulation, (Figure 7a), and the output is sma11. It goes to zero and reverses its phase at this potential (Figure $7 \mathrm{~b}$ ) and becomes in phase with the modulation beyond it (Figure 7c). The amplitude itself sharply increases with the probe potential, and has a broad maximum as shown in Figure 8. This is explained qualitatively in the following way; let the current voltage characteristic of the probe be of the form

$$
\begin{aligned}
& I=f(V), \text { and the applied voltage to the probe } \\
& V=V_{0}+V_{1}\left(1+\alpha \sin \omega_{2} t\right) \sin \omega_{1} t
\end{aligned}
$$

where

$$
\begin{aligned}
& \mathrm{V}_{0}: \text { DC voltage } \\
& \mathrm{V}_{1}: \text { RF voltage } \\
& \alpha: \text { ratio of modulation } \\
& w_{1}: \text { carrier RF frequency } \\
& w_{2}: \text { audio frequency of modulation }
\end{aligned}
$$

Then the obtained audio output is given by $A=\frac{1}{\tau} \int_{t}^{t+\tau} f(V) d \tau$

$=f\left(v_{0}\right)+\frac{1}{4} f^{\prime \prime}\left(v_{0}\right) v_{1}^{2} \cdot \alpha \cdot \sin w_{2} t+. \cdot$.

where $T$ is chosen much larger than the period of the RF wave. As can be seen from this equation, the audio output reverses its phase at $f^{\prime \prime}\left(V_{o}\right)=0$. The dependence of the audio output on the $R F$ voltage and the ratio of modulation is shown in Figures 9 and 10. The audio output increases sharply with the RF voltage, while it increases linearly with the ratio of modulation, just as the equation (1) predicts.

\section{DISCUSSION}

The detection of audio waves from radio stations by rockets is explained in a manner similar to the behavior of the ordinary resonance probe. Futhermore, this technique offers 
a useful method for plasma diagnostics which has several advantages over the ordinary resonance probe from practical points of view. First, the $\mathrm{S} / \mathrm{N}$ ratio is improved owing to the $\mathrm{AC}$ measurement, in general. Second, in the ordinary resonance probe the probe potential is set below the potential at which the audio output shows the phase reversal with respect to the modulation. However as can be seen from Figure 8 , a much larger output can be obtained at higher probe potential. The ordinary resonance probe is very sensitive to the probe-to-plasma potential and the resonance signal is soon masked by a large DC current when the probe potential is raised above the space potential. When the modulation method described above is employed, the large DC current does not contribute to the measurement, and a much larger output can be obtained for a larger plasma-to-probe potential. In rocket and satellite measurements in the ionosphere and outer space, we cannot control the rocket-to-plasma potential, and this method might be quite useful.

It is quite interesting to study the non-linear phenomena of the plasma and there have been several experiments on this problem by means of laser and microwaves (Chang et al, 1966; Ramsden et al, 1966; Weichel et al, 1967), most of which are for a higher density plasma. For such low density plasmas as used in this experiment, the non-linearity of the sheath around the electrodes is so large that it masks the non-linearity of the plasma itself. The effect of the sheath is inevitable in this type of low density plasmas. When we increase the RF voltage, in order to enhance the non-linear effect, the problem of ionization of residual gases also arises. Therefore, it is quite difficult to see non-linear phenomena of plasma itself such as the coupling between electron plasma waves and ion waves, and there have been no experiments for low density plasmas which show the non-linear phenomena of a plasma. Since the plasma 
which is used in this experiment is very quiet, and it represents a good medium for the study of linear phenomena in plasma physics, our future experiments will be concerned with the detection of the non-linearity of the plasma itself.

The author wishes to express his sincere thanks to Dr. J. A. Kane, who kindly offered his experimental facilities and is also grateful for his valuable discussions. He is also grateful to Dr. S. J. Bauer for his hospitality and encouragement during the course of the study.

The present work was carried out while the author held a postdoctoral resident research associateship of the NAS-NAE at the NASA-Goddard Space Flight Center, on leave of absence from the Japan Atomic Energy Research Institute, Ibaraki, Japan. 


\section{REFERENCES}

Butler, H. S., and G. S. Kino, (1963). Plasma Sheath Formation by Radio Frequency Fields. Phys. Fluids 6, 1346-1355

Chan, P. W. and R. A. Nodwe11 (1966). Collective Scattering of Laser Light by a Plasma. Phys. Rev. Letters 16, 122 Dote, T. and T. Ichimiya (1965). Characteristics of Resonance Probes. J. Appl Phys. 36, 1866-1872

Maynard, N. C., (1967). At the 48th annual meeting of the American Geophysical Union, Wahsington, D.C., 1967 (Transaction AGU 48, 156)

Ramsden, S. A., and W. E. R. Davis, (1966). Observation of Cooperative Effects in the Scattering of a Laser Beam from a Plasma. Phys. Rev. Letters 16,303

Takayama, K., H. Ikegami, and S. Miyazaki, (1960). Plasma resonance in a radio frequency probe, Phys. Rev. Letters $\underline{5}$, 238

Ungstrup, U., (Private Communication)

Weiche1, H., P. V. Avisonis and D. F. Vonderhaar, (1967). Observation of Plasma Ion Oscillations in a Laser-Produced Plasma. Phys. Rev. Letters 19, 10 


\section{FIGURE CAPTIONS}

Figure 1. Schematic layout of experiment.

Figure 2. Upper: audio output from the receiving electrode $P_{2}$ at resonance $(0.005 \mathrm{~V} / \mathrm{div})$

Lower: amplitude-modulated input $R F$ ( $5 \mathrm{~V} / \mathrm{div}$ )

Resonance frequency $=13.8 \mathrm{Mc} / \mathrm{s}$

Modulation frequency $=5 \mathrm{kc} / \mathrm{s}(50 \mu \mathrm{sec} / \mathrm{div})$

Figure 3. Dependence of audio output on the carrier frequency. Modulation frequency $=5 \mathrm{kc} / \mathrm{s}$, and 20 percent modulation.

Figure 4. Dependence of the phase $1 \mathrm{ag}$ from $180^{\circ}$ with respect to the modulation and the amplitude of the audio output on the modulation frequency.

Resonance Frequency $=15 \mathrm{Mc} / \mathrm{s}, 40$ percent modulation.

Figure 5. Dependence of the phase lag of the audio output from $180^{\circ}$ with respect to the modulation and the amplitude of the audio output on the external resistor.

Resonance frequency $=10.7 \mathrm{Mc} / \mathrm{s}$

Modulation frequency $=5 \mathrm{kc} / \mathrm{s}$.

Figure 6. Comparison of the frequency for the maximum audio output with the resonance frequency of the ordinary resonance probe, when the probe $P_{2}$ is used as a resonance probe.

$f_{R F}$ : frequency for the maximum audio output

$f_{\text {res }}$ : resonance frequency of the ordinary resonance probe. 
Figure 7. Oscilloscope traces showing the reversal of the relative phase of the audio output to the input modulation depending on the probe potential to the ground, $v_{0}$.
(a) $\mathrm{v}_{\mathrm{o}}=5 \mathrm{~V}(0.02 \mathrm{~V} / \mathrm{div})$
(b) $10(0.01 \mathrm{~V} / \mathrm{div})$
(c) $30(0.2 \mathrm{~V} / \mathrm{div})$
(d) Input RF (5V/div)

Resonance frequency $=10.7 \mathrm{Mc} / \mathrm{s}$ and modulation frequency $=200 \mathrm{c} / \mathrm{s}(2 \mathrm{msec} / \mathrm{div})$

Figure 8. Dependence of the amplitude of the audio output on the probe potential to the ground, $\mathrm{V}_{\mathrm{O}}$. Resonance frequency $=10 \mathrm{Mc} / \mathrm{s}$ and modulation frequency $=200 \mathrm{c} / \mathrm{s}$.

Figure 9. Dependence of the audio output on the RF voltage for various modulation frequencies. Resonance frequency $=10 \mathrm{Mc} / \mathrm{s}$, and 10 percent modulation .

Figure 10. Dependence of the audio output on the ratio of modulation. Resonance frequency $=10 \mathrm{Mc} / \mathrm{s}$ and modulation frequency $=500 \mathrm{c} / \mathrm{s}$. 


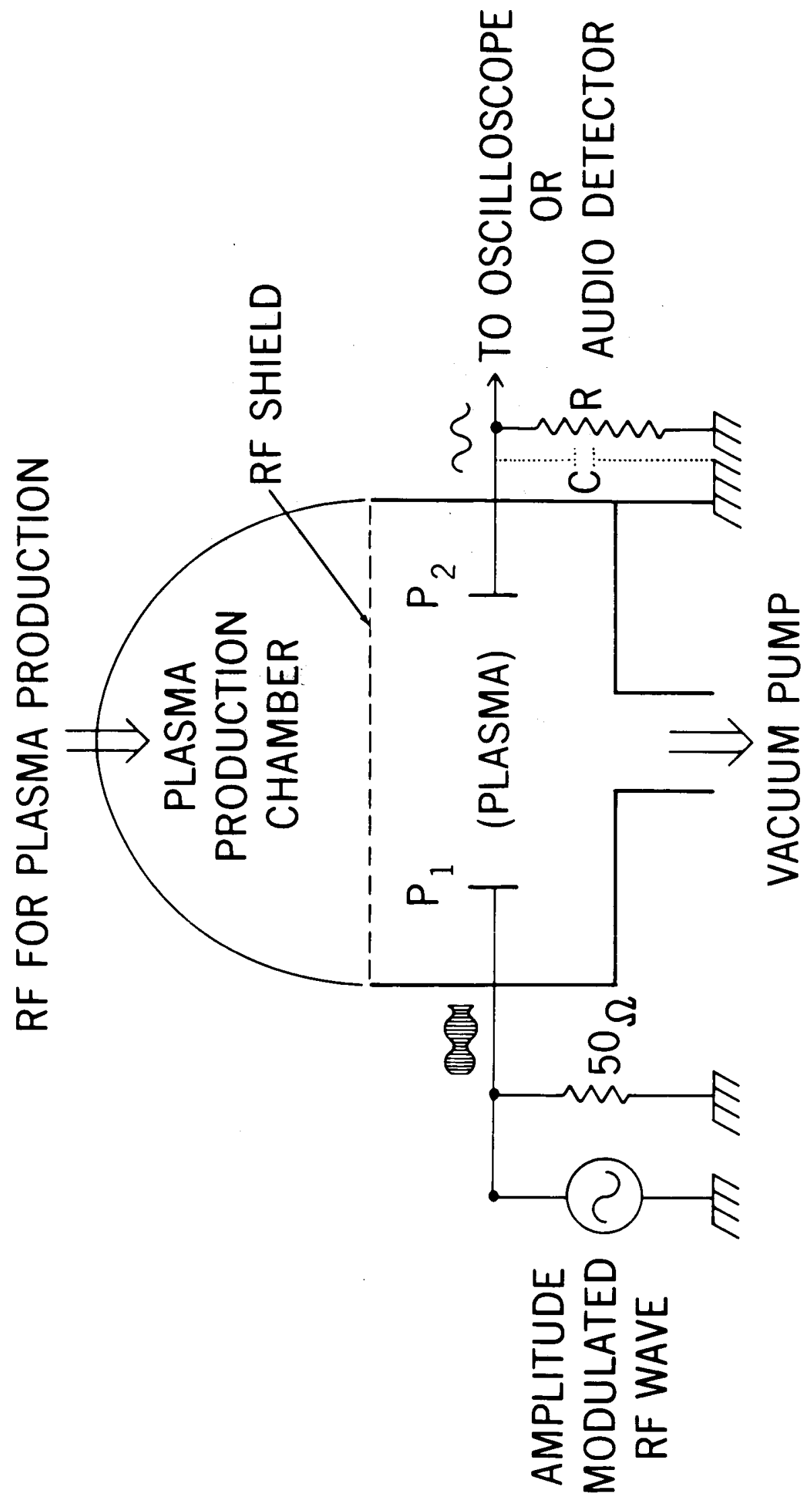

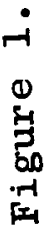




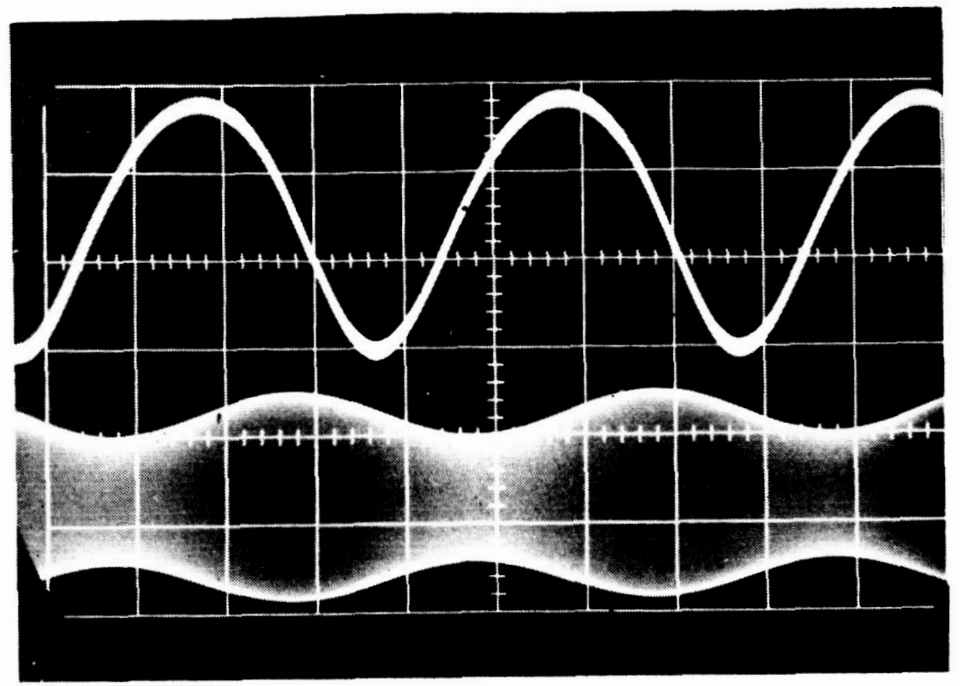

Figure 2. 


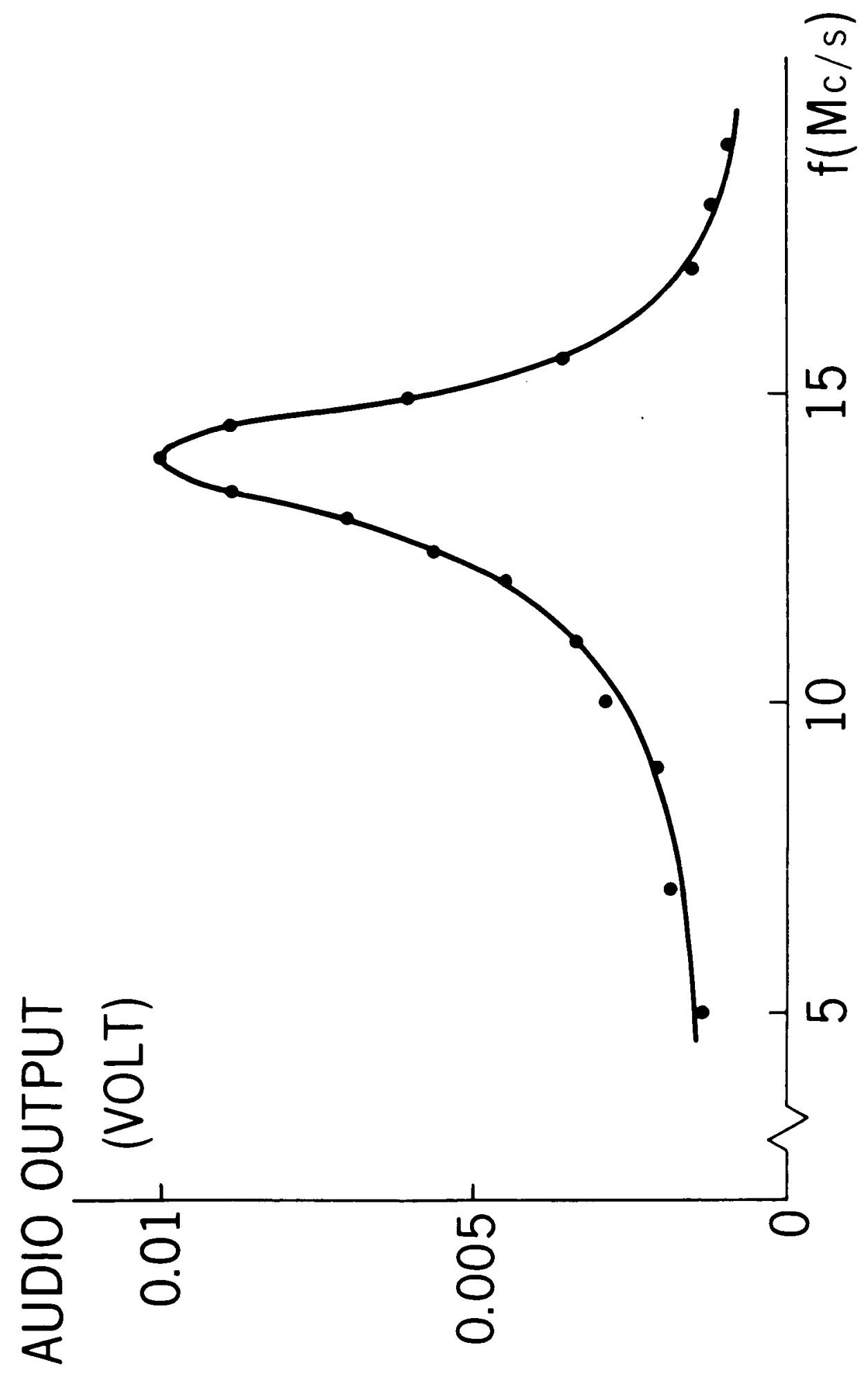

i 

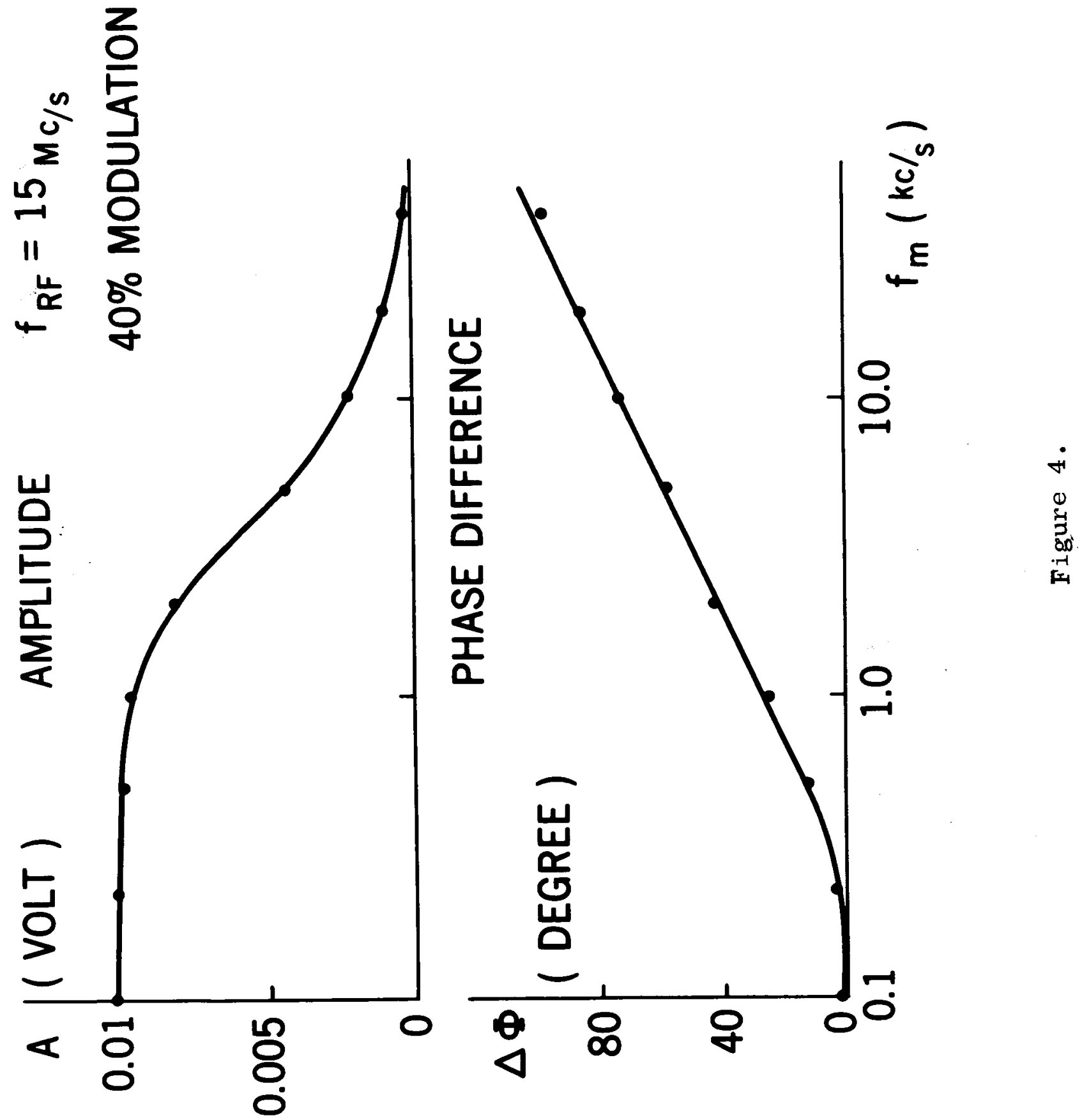


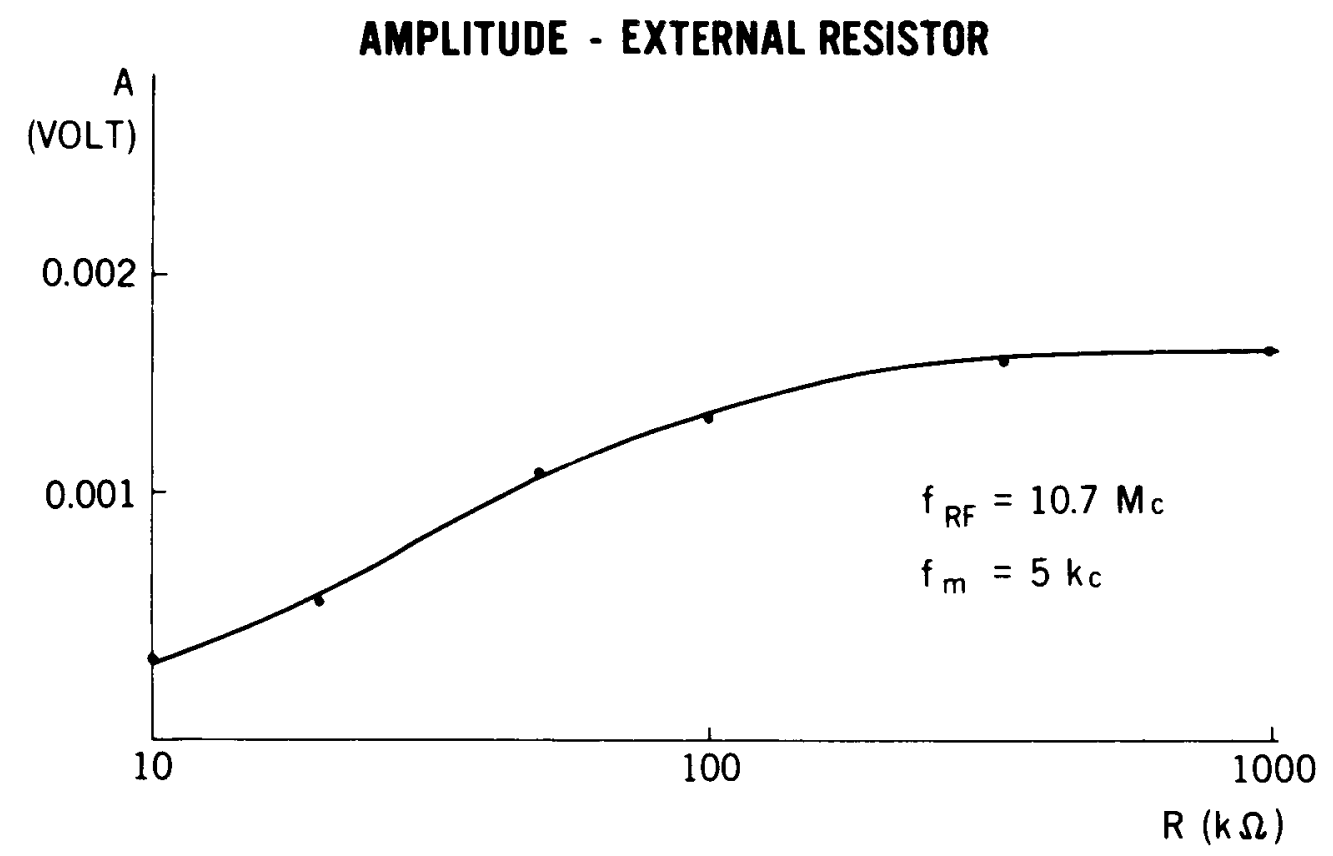

PHASE DIFFERENCE - EXTERNAL RESISTOR

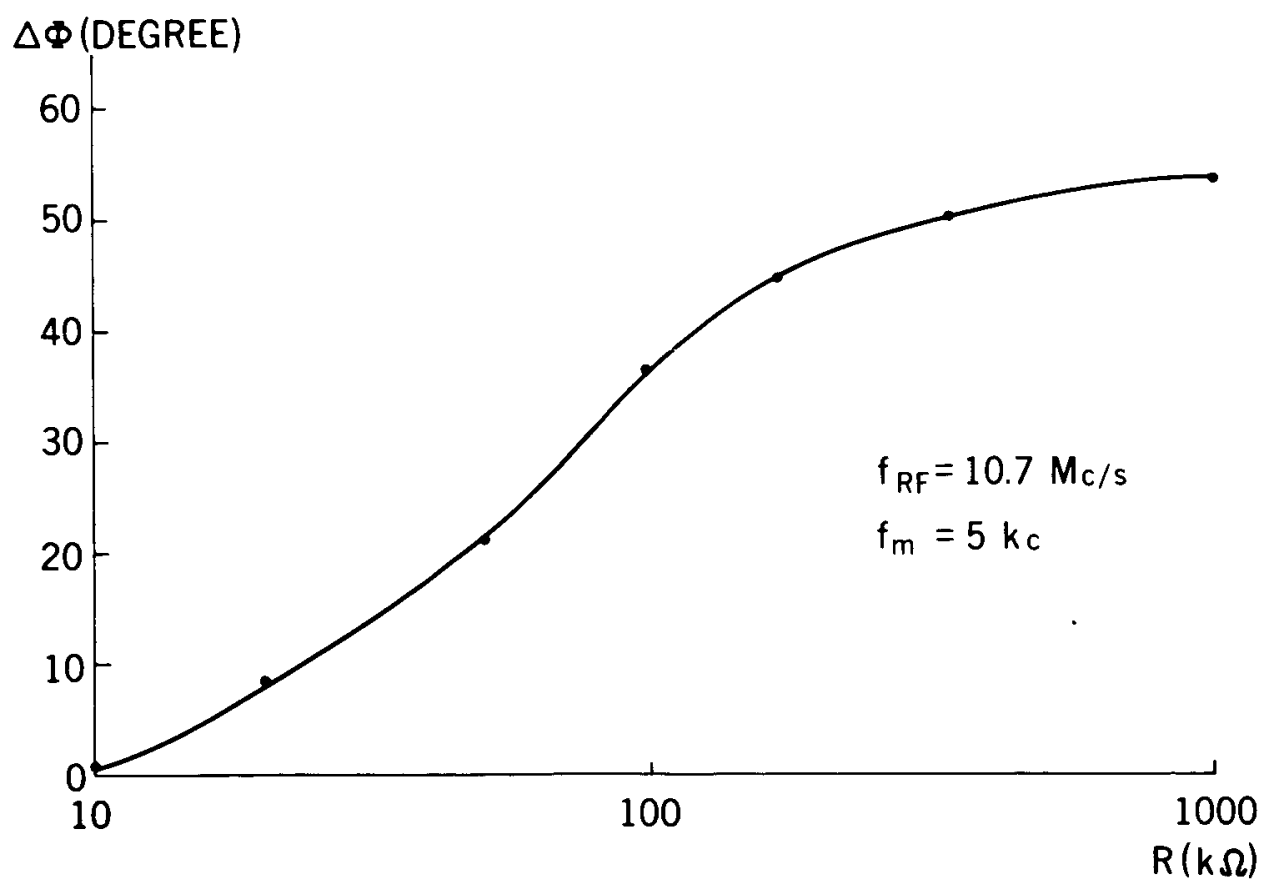

Figure 5. 


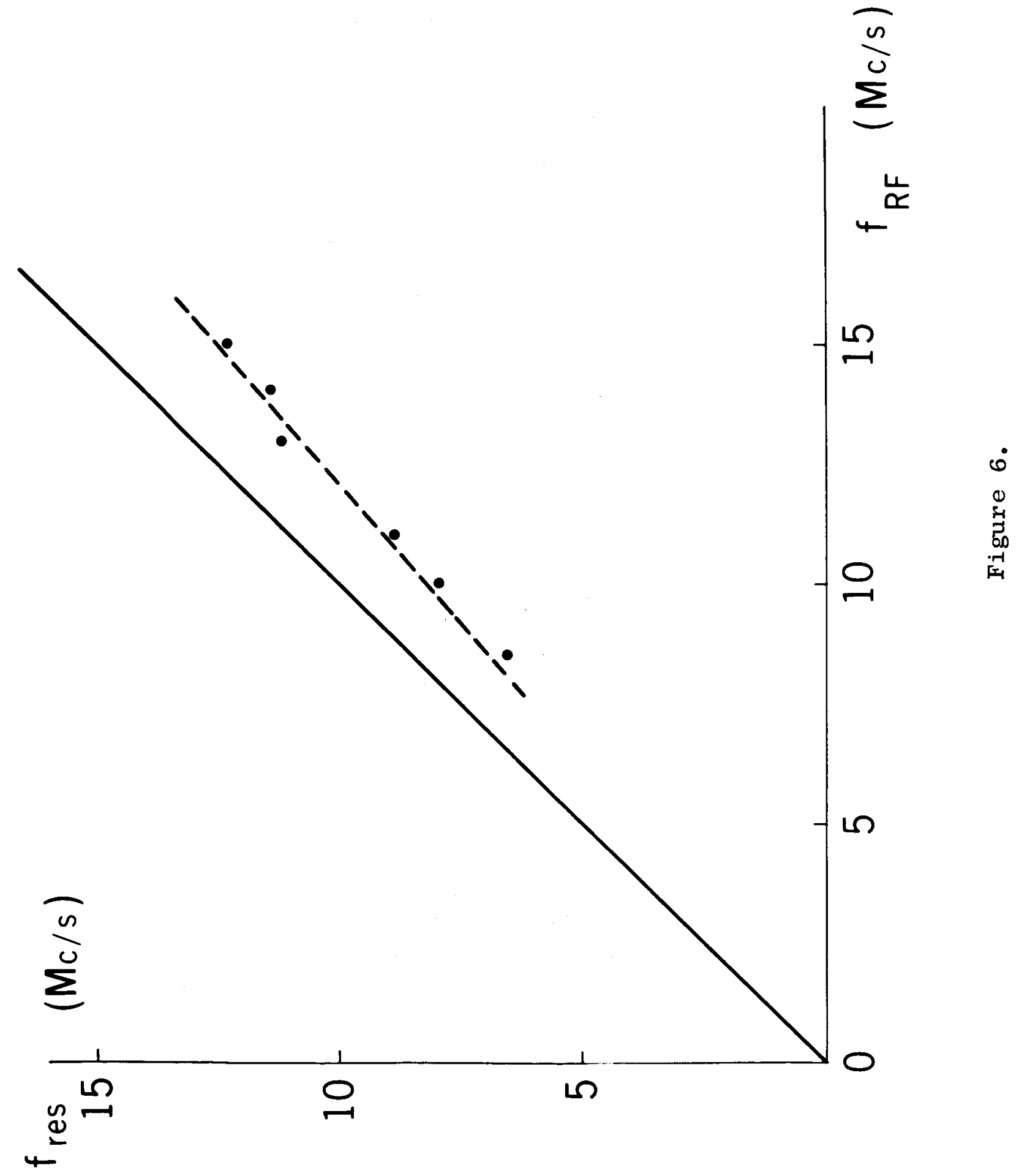


(a)

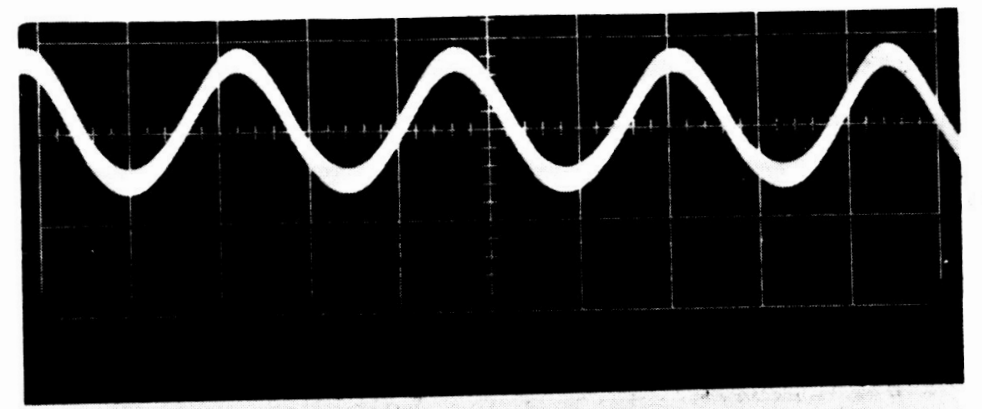

(b)
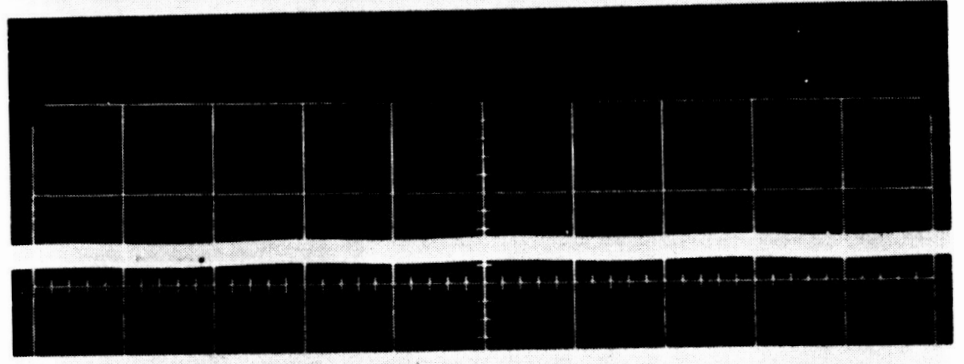

(c)

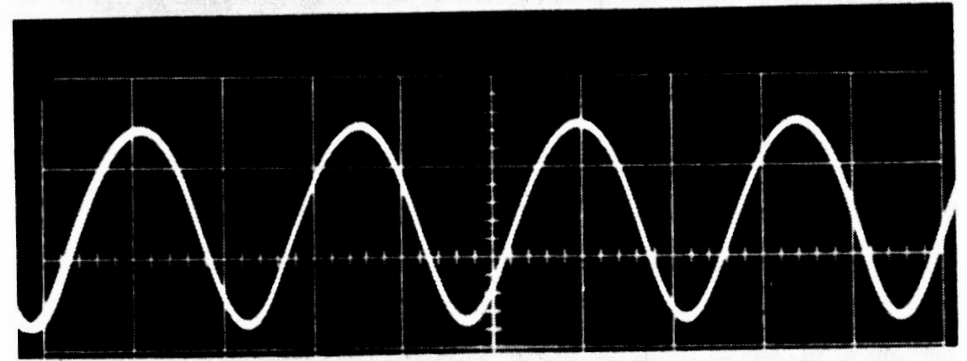

(d)

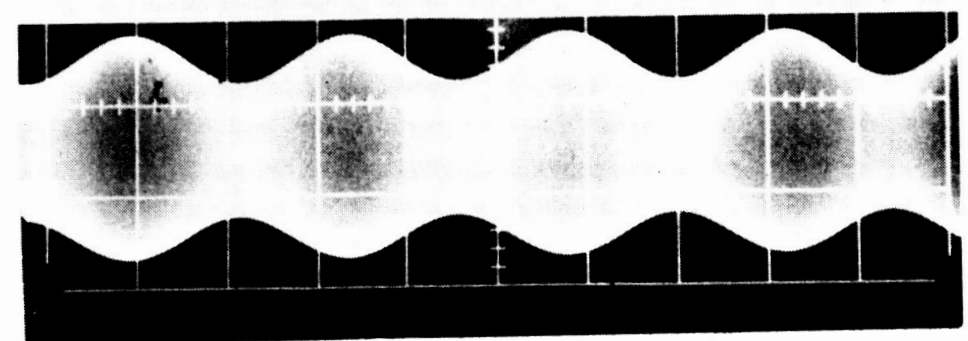

Figure 7 . 


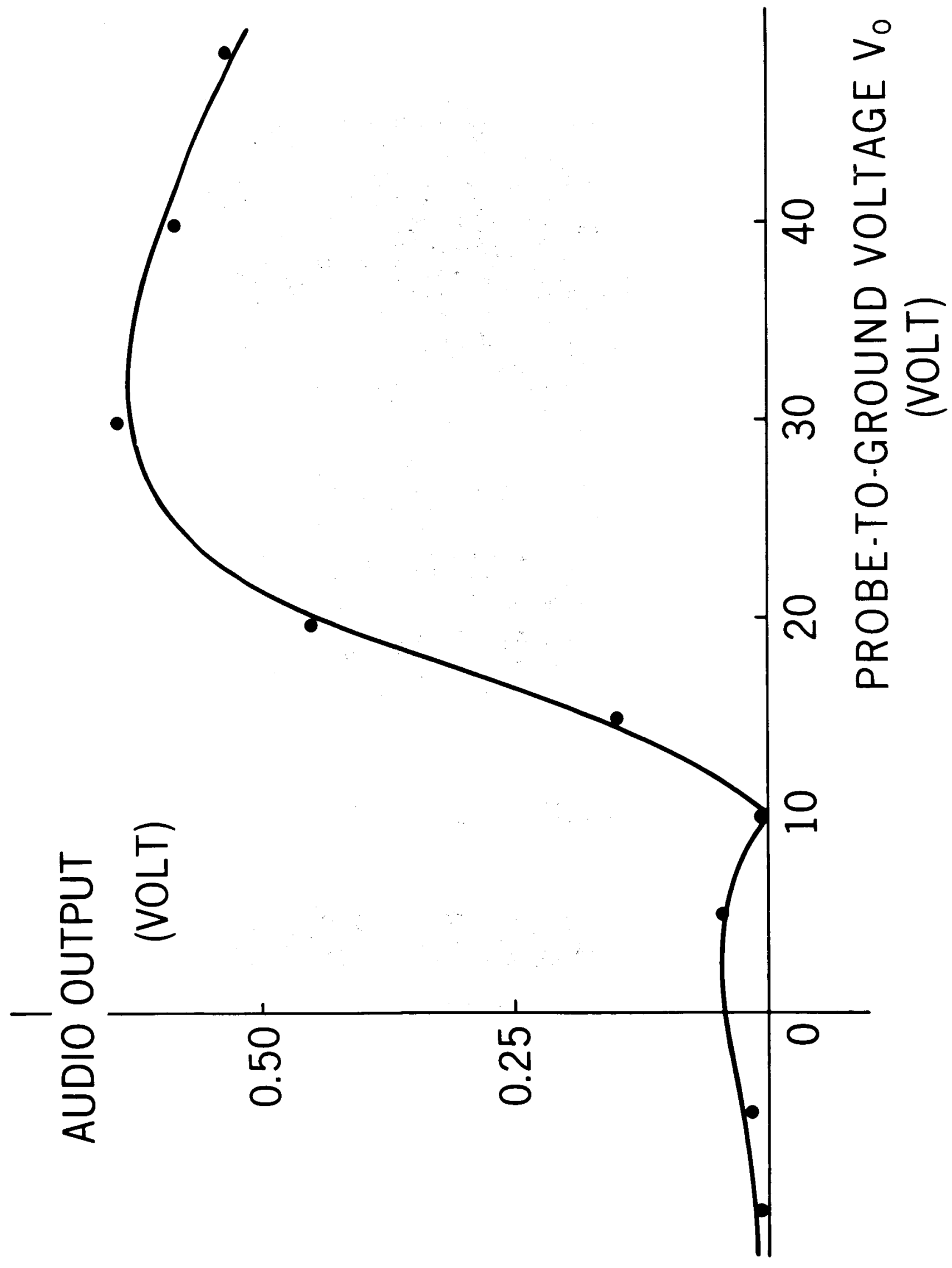




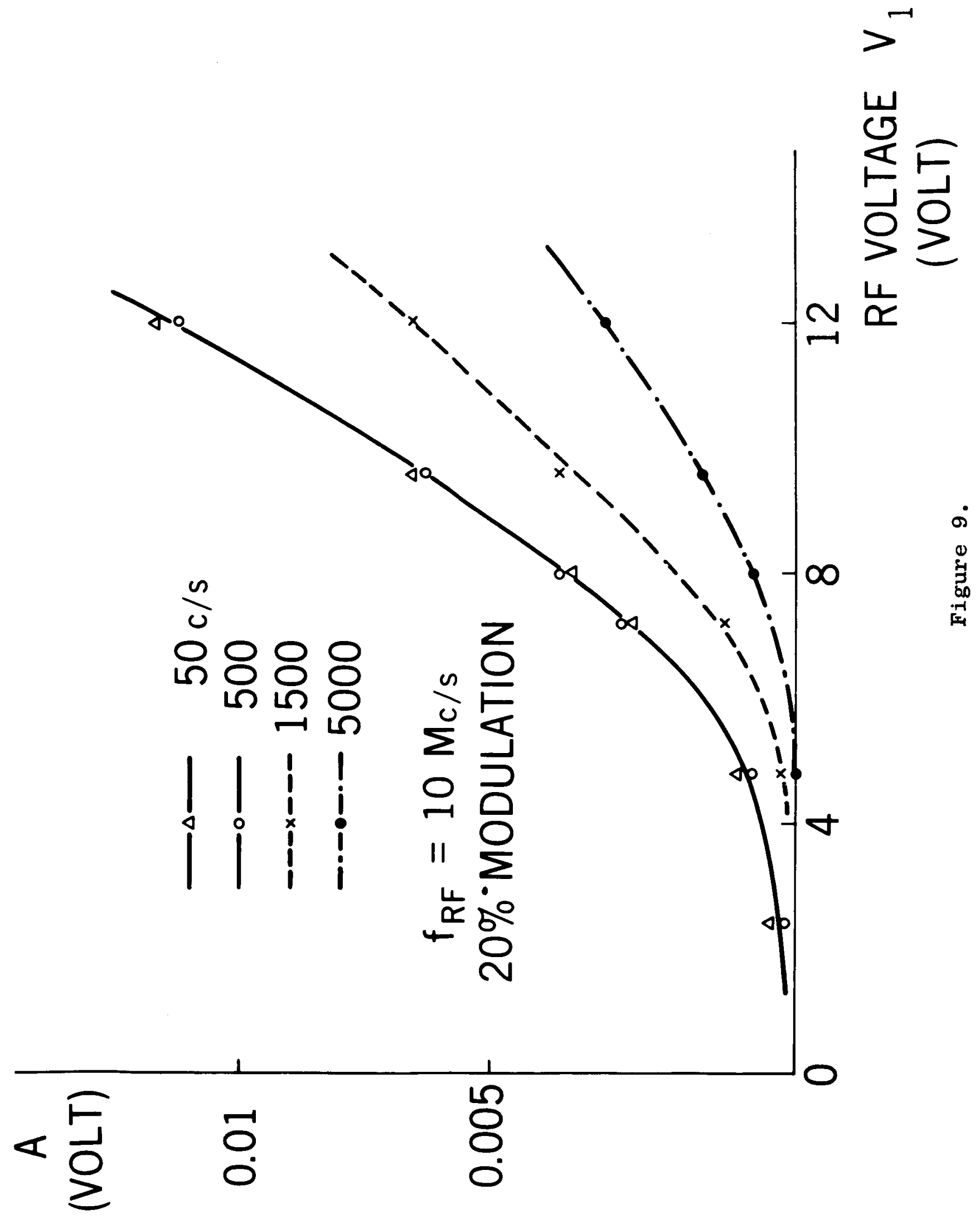




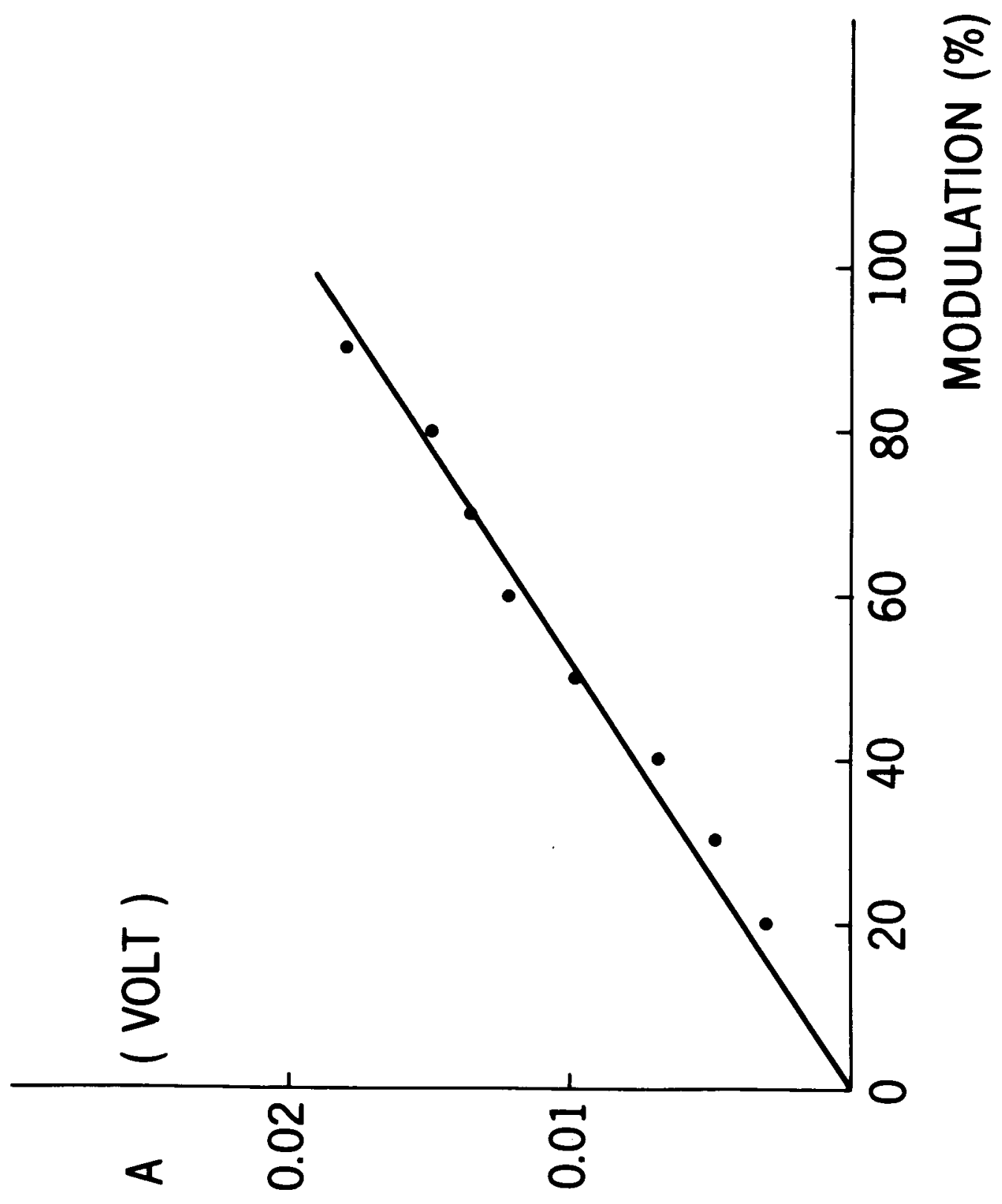

0
0
0
0
$y$
5
50
:
E 